\title{
Accuracy and practical aspects of semi- and fully automatic segmentation methods for resected brain areas
}

\author{
Karin Gau ${ }^{1}$ (1) $\cdot$ Charlotte S. M. Schmidt ${ }^{1,2} \cdot$ Horst Urbach $^{3} \cdot$ Josef Zentner $^{4} \cdot$ Andreas Schulze-Bonhage $^{1}$. \\ Christoph P. Kaller ${ }^{2,3} \cdot$ Niels Alexander Foit ${ }^{2,4}$
}

Received: 26 January 2020 / Accepted: 14 June 2020 / Published online: 20 July 2020

(C) The Author(s) 2020

\begin{abstract}
Purpose Precise segmentation of brain lesions is essential for neurological research. Specifically, resection volume estimates can aid in the assessment of residual postoperative tissue, e.g. following surgery for glioma. Furthermore, behavioral lesion-symptom mapping in epilepsy relies on accurate delineation of surgical lesions. We sought to determine whether semi- and fully automatic segmentation methods can be applied to resected brain areas and which approach provides the most accurate and cost-efficient results. Methods We compared a semi-automatic (ITK-SNAP) with a fully automatic (lesion_GNB) method for segmentation of resected brain areas in terms of accuracy with manual segmentation serving as reference. Additionally, we evaluated processing times of all three methods. We used T1w, MRI-data of epilepsy patients $(n=27 ; 11 \mathrm{~m}$; mean age 39 years, range 16-69) who underwent temporal lobe resections (17 left).

Results The semi-automatic approach yielded superior accuracy $(p<0.001)$ with a median Dice similarity coefficient $(\mathrm{mDSC})$ of 0.78 and a median average Hausdorff distance (maHD) of 0.44 compared with the fully automatic approach (mDSC 0.58 , maHD 1.32). There was no significant difference between the median percent volume difference of the two approaches $(p>0.05)$. Manual segmentation required more human input (30.41 min/subject) and therefore inferring significantly higher costs than semi- (3.27 min/subject) or fully automatic approaches (labor and cost approaching zero).

Conclusion Semi-automatic segmentation offers the most accurate results in resected brain areas with a moderate amount of human input, thus representing a viable alternative compared with manual segmentation, especially for studies with large patient cohorts.
\end{abstract}

Keywords Segmentation $\cdot$ Accuracy $\cdot$ Epilepsy surgery $\cdot$ Temporal lobe

Karin Gau

karin.gau@gmail.com

1 Epilepsy Center, Medical Center - University of Freiburg, Faculty of Medicine, University of Freiburg, Breisacher Str. 64, 79106 Freiburg im Breisgau, Germany

2 Freiburg Brain Imaging, Medical Center - University of Freiburg, Faculty of Medicine, University of Freiburg, Freiburg im Breisgau, Germany

3 Department of Neuroradiology, Medical Center - University of Freiburg, Faculty of Medicine, University of Freiburg, Freiburg im Breisgau, Germany

4 Department of Neurosurgery, Medical Center - University of Freiburg, Faculty of Medicine, University of Freiburg, Freiburg im Breisgau, Germany

\section{Introduction}

Studying associations between structural brain lesions and observable functional deficits constitutes a well-established approach in neuroscience research [1-3]. Quantitative analysis techniques such as voxel-based lesion-symptom mapping (VLSM) [1, 4] or overlap calculations between lesions and critical structures [5-7] are employed to link structural alterations to behavioral markers [8], hence detecting brain areas critical for a specific behavior. Precise determination of lesioned tissue components in neuroimaging data therefore constitutes a crucial step for these methods $[5,9,10]$.

To date, slice-by-slice manual lesion tracing by expert raters remains the gold standard $[5,11,12]$. This approach is considered most precise [3], but also tedious and timeconsuming [13] and requires significant experience [14]. Therefore, lesion segmentation constitutes a significant cost factor in neuroimaging research. Semi-automatic methods 
seek to overcome these limitations $[15,16]$. Generally, their approach is based on the assumption that alterations in tissue homogeneity [17], composition, shape [18], or laterality [19] represent lesioned tissue and their identification would only require fine-tuning of parameters by a supervisor [20]. Despite being less laborious than manual tracing, significant amounts of user-interaction are nevertheless required [20].

Recently, several fully automatic methods for lesion segmentation using machine learning (ML) $[17,21,22]$ approaches have been proposed. Briefly, ML allows for the design of algorithms that can learn from training datasets and make predictions on new data. ML approaches are usually classified into two broad categories, namely supervised and unsupervised learning [23]. In supervised learning, manually labeled training data is fed to the algorithm to enable categorization of new data-supervision is provided during training [21]. Conversely, unsupervised approaches rely on the recognition of latent patterns within the data to identify groups or clusters [13, 24-28].

In temporal lobe epilepsy (TLE), precise lesion delineation can inform surgical planning [29] and facilitate research on cognitive outcomes [30,31]. While VLSM constitutes a core component of stroke research [1], its application in TLE has been limited to date. However, with the introduction of selective procedures such as laser amygdalohippocampectomy [32], VLSM in TLE now receives growing interest [7], specifically for the study of memory impairment $[33,34]$. Additionally, assessment of lesion volume data can significantly improve image registration and normalization to stereotaxic space $[35,36]$. Furthermore, postoperative resection volumes are particularly useful for the assessment of completeness of resection [29] and seizure outcome prediction [37]. Clearly, there is a need to obtain accurate resection volumes from imaging data in TLE. However, to date, no segmentation algorithm specifically designed to investigate neurosurgical resections has been proposed.

In the present study, we therefore sought to determine;

a) whether supervised semi- and fully automatic algorithms each provide satisfying accuracy in resected brain areas compared with manual segmentation;

b) which of the three methods is most time- and costefficient and offers the best ratio between quality and cost.

\section{Methods}

\section{Subjects and imaging data}

This retrospective study was approved by the local institutional review board and individual informed consent was waived. High-resolution, T1-weighted magnetization-prepared rapid gradient echo datasets from 27 TLE patients after unilateral anterior temporal lobectomy (ATL) or selective amygdalohippocampectomy (sAHE) acquired with a standard 32-channel headcoil (MPRAGE; TR $=2200 \mathrm{~ms}$; TE = $2.15 \mathrm{~ms}$; flip angle $=12^{\circ} ; 160-176$ sagittal slices, voxel size $1 \times 1 \times 1 \mathrm{~mm}^{3}$ ) on a 3-T TIM Trio clinical MRI scanner (Siemens Healthineers AG, Erlangen, Germany) were retrieved from PACS (Picture Archiving and Communication System). Postoperative imaging was performed during routine follow-up median 3 months after epilepsy surgery (range 122 months). Clinical and demographic data of the study group are summarized in Table 1. All statistical analyses were performed with SPSS version 23.0 (IBM, Chicago, IL).

\section{Preprocessing}

All imaging data preprocessing was performed with statistical parametric mapping software (SPM version 12, fil.ion.ucl.ac. uk) running in MATLAB R2016b (The Mathworks, Nattick, USA). After visual inspection of image quality, all $\mathrm{T} 1 \mathrm{w}$ images with right hemispheric lesions were flipped to align all pathology to the left.

\section{Computational platform}

Manual, semi-automatic, and fully automatic segmentation was performed on a 3.2-GHz Intel Core i7-6600U platform with 12-GB RAM under Windows 10. MATLAB R2016b and SPM12 were used to run the GNB classifier.

\section{Manual segmentation}

Hand-drawn resection area maps served as ground truth [20]. Delineation was performed by an experienced rater (CS, neuroscience researcher with more than 5 years of experience in manual lesion delineation) blinded to the results of other segmentation methods. The boundaries of the resection areas were hand-drawn on consecutive axial slices with MRIcron (www.mricro.com) in patient space and then automatically filled, resulting in a three-dimensional region of interest (ROI).

\section{User-guided, semi-automatic segmentation}

Semi-automatic segmentation was performed with ITKSNAP toolbox version 3.6 (further referred to as SNAP) [16]. The "region competition" segmentation approach as well as the active contour evolution algorithm and their implementation in the ITK-SNAP has been previously described in detail $[16,38]$. Briefly, the rater defines a segmentation domain to restrict the algorithm to a volumetric ROI. For pre-segmentation, we used the soft-thresholding intensity-based mode [39]: By manually applying a two-sided threshold depending on the intensity range of the ROI (foreground), an intensity grading vector "speed image" is generated to broadly define 
Table 1 Demographic data

\begin{tabular}{|c|c|c|c|c|c|c|c|c|}
\hline Patient & Gender & $\begin{array}{l}\text { Age at } \\
\text { surgery }\end{array}$ & $\mathrm{AoO}$ (years) & Duration (years) & $\begin{array}{l}\text { Side of } \\
\text { surgery }\end{array}$ & Type of surgery & Pathology & $\begin{array}{l}\text { ILAE seizure } \\
\text { outcome-1 year }\end{array}$ \\
\hline 1 & $\mathrm{~F}$ & 34 & 0.75 & 33.25 & $\mathrm{~L}$ & ATL & Dual (FCD) & $3 a$ \\
\hline 2 & M & 20 & 12 & 8 & $\mathrm{~L}$ & ATL & Dual (FCD) & $2 \mathrm{~b}$ \\
\hline 3 & M & 53 & 9 & 44 & $\mathrm{~L}$ & ATL & Dual (FCD) & $3 a$ \\
\hline 4 & $\mathrm{~F}$ & 69 & 17 & 52 & $\mathrm{~L}$ & ATL & FCD & $3 b$ \\
\hline 5 & $\mathrm{~F}$ & 32 & 7 & 25 & $\mathrm{~L}$ & ATL & FCD & 1a \\
\hline 6 & $\mathrm{~F}$ & 56 & 13 & 43 & $\mathrm{~L}$ & ATL & Dual (FCD) & $1 \mathrm{a}$ \\
\hline 7 & M & 38 & 17 & 21 & $\mathrm{R}$ & ATL & HC sclerosis & $2 \mathrm{~b}$ \\
\hline 8 & M & 50 & 14.0 & 36 & $\mathrm{~L}$ & ATL & Dual (FCD) & $1 b$ \\
\hline 9 & $\mathrm{~F}$ & 16 & 0.5 & 15.5 & $\mathrm{~L}$ & ATL & Dual (FCD) & 1a \\
\hline 10 & M & 53 & 39 & 14 & $\mathrm{R}$ & ATL & $\mathrm{HC}$ sclerosis & $2 b$ \\
\hline 11 & M & 20 & 10 & 10 & $\mathrm{R}$ & ATL & HC sclerosis & 1a \\
\hline 12 & $\mathrm{~F}$ & 28 & 7 & 21 & $\mathrm{~L}$ & ATL & HC sclerosis & $1 \mathrm{a}$ \\
\hline 13 & M & 25 & 21 & 4 & $\mathrm{R}$ & ATL & Dual (FCD) & $1 \mathrm{a}$ \\
\hline 14 & $\mathrm{~F}$ & 55 & 32 & 23 & $\mathrm{R}$ & sAHE & HC sclerosis & $1 \mathrm{a}$ \\
\hline 15 & $\mathrm{~F}$ & 47 & 3 & 44 & $\mathrm{~L}$ & sAHE & $\mathrm{HC}$ sclerosis & $3 a$ \\
\hline 16 & M & 47 & 26 & 21 & $\mathrm{~L}$ & sAHE & HC sclerosis & 1a \\
\hline 17 & $\mathrm{~F}$ & 46 & 30 & 16 & $\mathrm{R}$ & sAHE & HC sclerosis & $2 \mathrm{~b}$ \\
\hline 18 & $\mathrm{~F}$ & 21 & 20 & 1 & $\mathrm{~L}$ & sAHE & Dual (ganglioglioma) & 1a \\
\hline 19 & $\mathrm{~F}$ & 42 & 32 & 10 & $\mathrm{~L}$ & sAHE & $\mathrm{HC}$ gliosis & $1 \mathrm{a}$ \\
\hline 20 & $\mathrm{~F}$ & 53 & 10 & 43 & $\mathrm{~L}$ & sAHE & $\mathrm{HC}$ sclerosis & $1 \mathrm{a}$ \\
\hline 21 & M & 22 & 15 & 7 & $\mathrm{R}$ & sAHE & HC sclerosis & $1 \mathrm{a}$ \\
\hline 22 & $\mathrm{~F}$ & 35 & 21 & 14 & $\mathrm{~L}$ & sAHE & HC sclerosis & $1 \mathrm{a}$ \\
\hline 23 & M & 22 & 19 & 3 & $\mathrm{R}$ & sAHE & $\mathrm{HC}$ sclerosis & 1a \\
\hline 24 & M & 48 & 2.0 & 46 & $\mathrm{~L}$ & sAHE & HC sclerosis & $1 \mathrm{a}$ \\
\hline 25 & $\mathrm{~F}$ & 39 & 3 & 36 & $\mathrm{R}$ & sAHE & $\mathrm{HC}$ sclerosis & $1 \mathrm{a}$ \\
\hline 26 & $\mathrm{~F}$ & 50 & 38 & 12 & $\mathrm{~L}$ & sAHE & HC sclerosis & 4 \\
\hline 27 & $\mathrm{~F}$ & 35 & 33 & 2 & $\mathrm{R}$ & sAHE & $\mathrm{HC}$ sclerosis & $1 \mathrm{a}$ \\
\hline Mean & $16 \mathrm{~F}$ & 39.1 & 16.7 & 22.4 & $17 \mathrm{~L}$ & & & $63 \%=1 \mathrm{a} ; 37 \%>1 \mathrm{a}$ \\
\hline Std. dev \pm & & 13.8 & 11.4 & 15.4 & & & & \\
\hline
\end{tabular}

$A o O$, age of onset; $F$, female; $M$, male; $L$, left; $R$, right; Dual, dual pathology; $F C D$, focal cortical dysplasia

lesion boundaries. In this speed image, intensity values between the lower and upper thresholds are assigned positive speed values and correspond to parts of the image that have higher probability of representing the ROI rather than the background. Values outside the thresholds map to negative speed values. To initiate geometric active contour segmentation, the rater places at least one seedpoint randomly inside the ROI, which will grow in a way that balances adherence to the speed image with a geometric regularization term [40]. The evolving contour is visualized in real time in $2 \mathrm{D}$ slices and evolves either with a fixed step size or continuously until manual termination by the user [41]. We chose to stop the algorithm if there was no further visible propagation in the image over 5 seconds or if the rater decided that the evolving segmentation began to leak outside the boundaries of the ROI.
This is a common approach other studies described before [42]. Finally, ROIs were inspected for quality and manually edited in two cases (shown in Fig. 1f): Firstly, if active contour segmentation bled into CSF space, manual editing was performed using the paintbrush tool. Secondly, if parts of the resected brain area, e.g., blood debris, were not identified as part of the ROI, we used the paintbrush with the interpolation module that allows to trace a structure in just a handful of slices, with the algorithm filling in the intermediate slices [43]. This segmentation method was used as it requires only minimal user-interaction and reliable results can be obtained from single-modality MRI. In this study, an expert rater (NAF, neuroscience researcher with more than 10 years of experience in lesion segmentation) performed the segmentation with ITK-SNAP blinded to the results of manual 

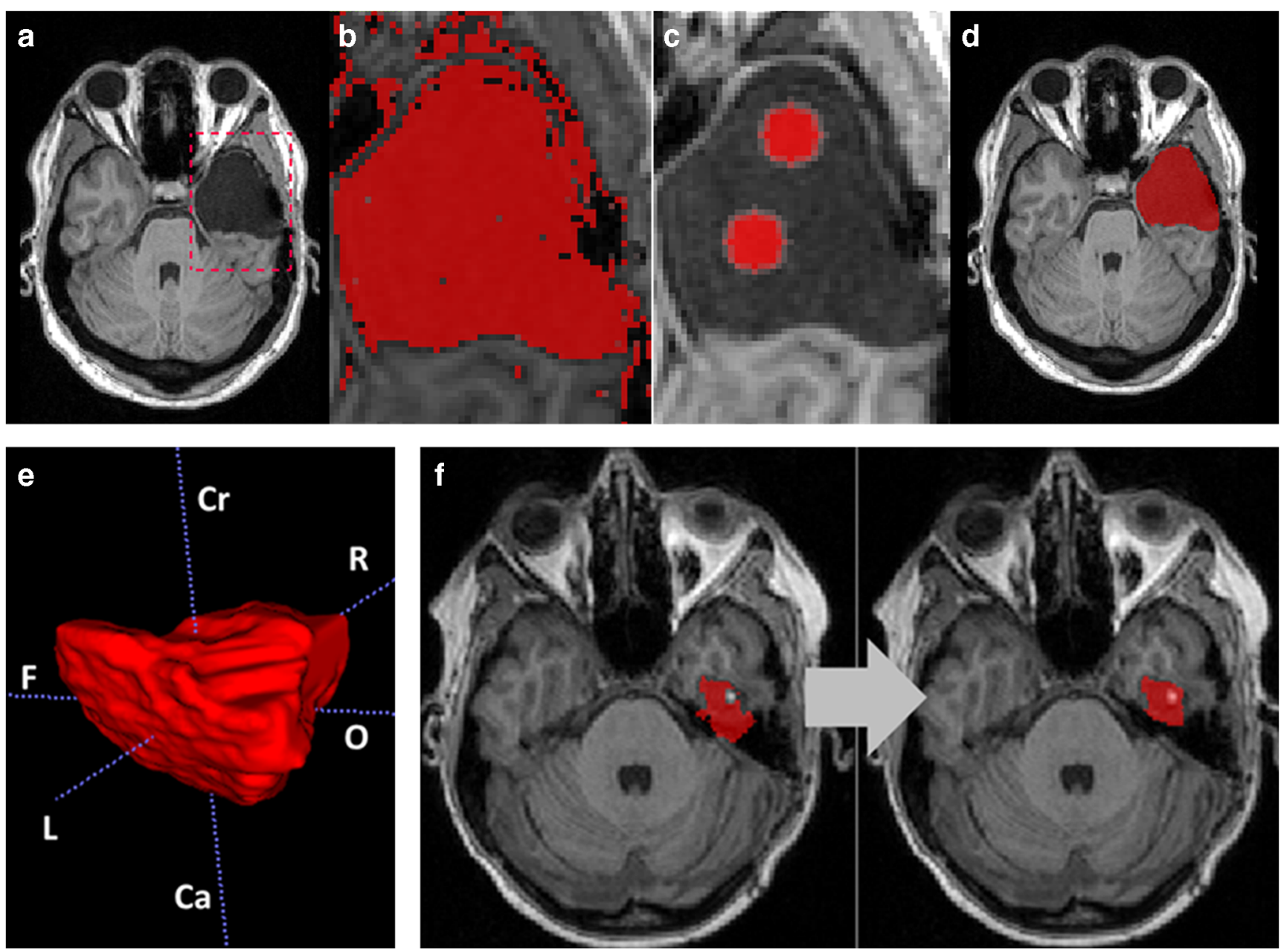

Fig. 1 ITK-SNAP segmentation workflow. a Region of interest definition. b Thresholding. c Placement of seedpoints. d Quality control. e Threedimensional polygonal lesion model $(\mathrm{F}=$ frontal; $\mathrm{O}=$ occipital; $\mathrm{L}=$ left; $\mathrm{R}=$ right; $\mathrm{Cr}=$ cranial; $\mathrm{Ca}=$ caudal $)$. $\mathbf{f}$ Manual editing

segmentation. Figure 1 illustrates individual steps of the semiautomatic segmentation process.

\section{Fully automatic segmentation}

The lesion_GNB software package by Griffis and coworkers [44] employs a supervised approach based on Gaussian Naïve Bayes (GNB) classification for the delineation of chronic stroke defect zones. To provide ground truth for classifier training, manual segmentation of T1w MRI data of 30 patients with chronic stroke defect zones was performed by the authors [44]. Briefly lesion_GNB relies on probabilistic tissue segmentation and image algebra to create feature maps encoding information about missing or abnormal tissue classes. The GNB classifier was trained on the ground-truth manually delineated lesions and validated using a leave-one-out crossvalidation approach. It does not require a control group. By using default SPM processing routines, transformation parameters for normalization to Montreal Neurological Institute (MNI) space are created during analyses [44].

\section{Spatial similarity analyses}

Most automated segmentation routines operate in algorithmspecific stereotaxic space, which may not correspond to MNI space [45]. However, studies investigating larger patient cohorts will ultimately require normalization to make group inferences. To avoid potential bias by comparing inversenormalized (lesion GNB) to native-space (manual, ITKsnap) results, we chose to perform spatial similarity analysis in common space. All ROIs were therefore normalized to standard MNI space with normalization parameters obtained during the lesion GNB preprocessing of individual subjects.

Degrees of overlap between the different lesion segmentation techniques referenced against manual tracing results were evaluated voxel-by-voxel with the Dice similarity coefficient (DSC), a robust metric of both overlap and reproducibility [46]. The DSC is calculated according to DSC $=\frac{2(\mathrm{X} \cap \mathrm{Y})}{(X+Y)}$. In case of partially overlapping image volumes, i.e., reference ( $X$; manual tracing) and predicted volume ( $Y$; semi-/fully automatic approach), the DSC ranges from 0 (no overlap) to 1 (total congruence), with 
larger values indicating better performance [42]. DSCs ranging above 0.6 are good, values above 0.7 are considered high, and values exceeding 0.8 are excellent $[13,20,44]$.

Although robust and straightforward to interpret, the DSC does not weigh false-positive or negative results in any way. This becomes particularly relevant in complex structures such as surgical lesions, where boundary agreement between segmentations might be of interest [47]. Therefore, we chose to supplement the analysis with a measure of spatial distance, i.e., the average Hausdorff distance (aHD). The aHD represents the maximum distance of all voxels in one dataset to corresponding voxels in the test set [48], thus quantifying similarity or discrepancy between two given structures [49]. The aHD between two point sets $\mathrm{X}$ and $\mathrm{Y}$ is defined as $\operatorname{aHD}(X, Y)=\max (d(X, Y), d(X, Y))$ where $d(X, Y)$ is the directed aHD that is given by $d(X, Y)=\frac{1}{N} \sum_{x \in X} \min _{y \in Y}\|x-y\|$ with reference ( $X$; manual tracing) and predicted volume ( $Y$; semi-/fully automatic approach). All distances are calculated in voxel with smaller values indicating better performance.

As the results were not normally distributed, they were compared using non-parametrical Wilcoxon signed-rank test, respectively. In order to assess the relation between performance of the approaches and the resection area size, a median split by lesion volume (based on manual segmentation) was performed. With a Mann-Whitney $U$ test, the difference of the metrics of the two groups (small/large lesions) within each approach was assessed and a Wilcoxon signed-rank test was used to compare the approaches within each group. Correlations were assessed with the non-parametrical Spearman's rank correlation.

\section{Volumetric analyses}

Percent lesion volume differences (in $\mathrm{ml}$ ) between manual and (semi-)automated segmentation results were also evaluated. Percent volume difference (PVD) was calculated according to $\mathrm{PVD}=\left[\frac{\text { Vreference- } \mathrm{V}(\text { semi-) automatic }}{\mathrm{V} \text { reference }}\right] \times 100$; with $V_{\text {reference }}$ and $V_{\text {(semi-)automatic }}$ representing volumes of manual and (semi-)automatic resection maps, respectively. Since resection volumes were not normally distributed, a Wilcoxon signedrank test was used for comparisons. We also assessed differences between large and small resections in the PVD within each approach with the Mann-Whitney $U$ test.

\section{Effort and cost}

The labor time (corresponding to human input) in minutes required from the beginning of the segmentation to saving the final ROI was recorded for each method. The time required for loading the image into each program was similar, so we did not take this step into account. As in the fully automatic approach no human input was necessary after loading the images, labor time therefore resulted in $0.00 \mathrm{~min} / \mathrm{sub}-$ ject. For manual and semi-automatic segmentation, the labor time was normally distributed, as assessed by the ShapiroWilk test $(p>0.05)$. The difference in time expenditure between the manual and semi-automatic approaches was compared with a paired-samples $t$ test. To compare the labor time of the manual and semi-automatic to the fully automatic approach $(0.00 \mathrm{~min} / \mathrm{subject})$, a one-sample $t$ test was used, respectively.

Because we only used open-source software and the available computational infrastructure, the total method cost in this study was mainly composed of personnel cost based on the salary rates of the German Research Foundation for 2019 (available at www.dfg.de/formulare).

\section{Results}

\section{Patient characteristic}

Data were acquired from 27 patients (16 females, 11 males), who underwent unilateral ATL (13 patients) or sAHE (14 patients) for pharmacoresistant TLE (17 left-sided, 10 rightsided). The mean age at surgery was 39.1 years (range 16-69), with a mean age at epilepsy onset of 16.7 years (range $0.5-39$ ) and a mean disease duration of 22.4 years (range 1-52). Approximately $63 \%$ of the patients were seizure-free (Engel IA) after surgery. The predominant pathology was hippocampal sclerosis (16 patients) followed by dual pathology (i.e., hippocampal sclerosis combined with focal cortical dysplasia; 8 patients). Detailed demographical and clinical data are given in Table 1.

\section{Accuracy}

Manual segmentation is the gold standard and serves as ground truth for the other methods. Figure 2 illustrates 27 manually segmented resection areas, overlaid on the respective T1w image.

The median DSC for the semi-automatic approach relative to the manual segmentation was 0.78 (range $0.53-0.94$ ), indicating a high overlap. For the fully automatic method, the median DSC was 0.58 (range 0.05-0.76). At $p<0.001(z=$ -4.332 ), the results of the semi-automatic approach were significantly better than those of the fully automatic (Fig. 3).

As shown in the right panel in Fig. 3, the fully automatic segmentation method performed worse in all cases except three, with several false positive results as shown in Fig. 4. The GNB classifier failed to identify one resection area (P16, DSC 0.05) and performed poorly in another two cases (P21, P22). Figure 5 shows the individual performance of all approaches in patient P16. Even if this case was excluded, there 


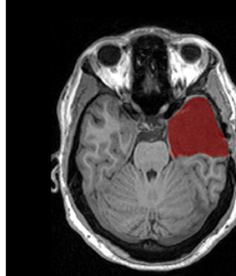

P 1

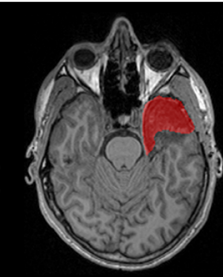

P 2

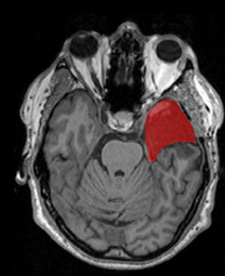

P 3

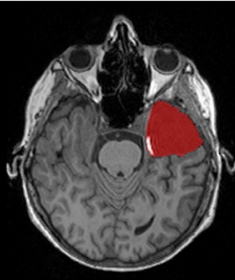

P 4

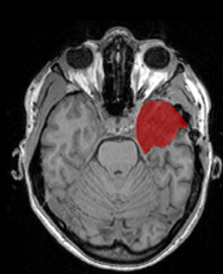

P 5

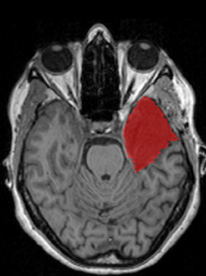

P 6



P 7

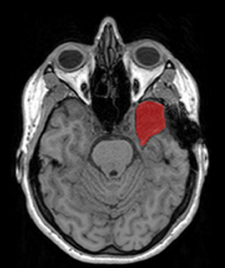

P 12

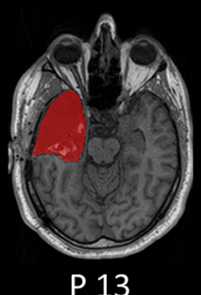

P 8

P 9

P 10

P 11

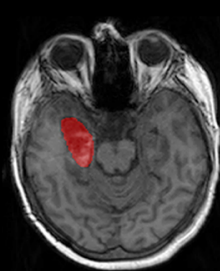

P 17



P 18

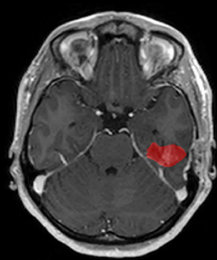

P 19

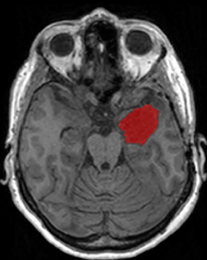

P 20



P 21

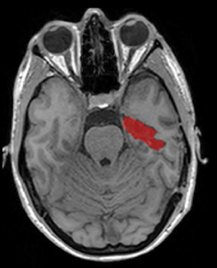

P 22

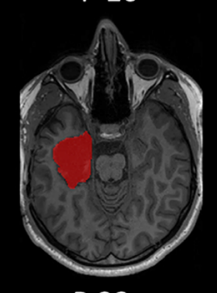

P 23



P 24

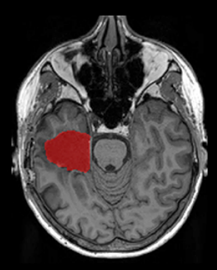

P 25

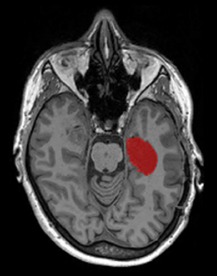

P 26



Fig. 2 Representative axial slices of the resected brain areas with superimposed manual segmentations (ATL: patients 1-13; sAHE: patients 14-27)

would still be a highly significant difference $(p<0.001, z=-$ 4.239) between the fully automatic (median DSC 0.60) and the semi-automatic (median DSC 0.79) approach. In terms of spatial distance, the semi-automatic approach (median aHD
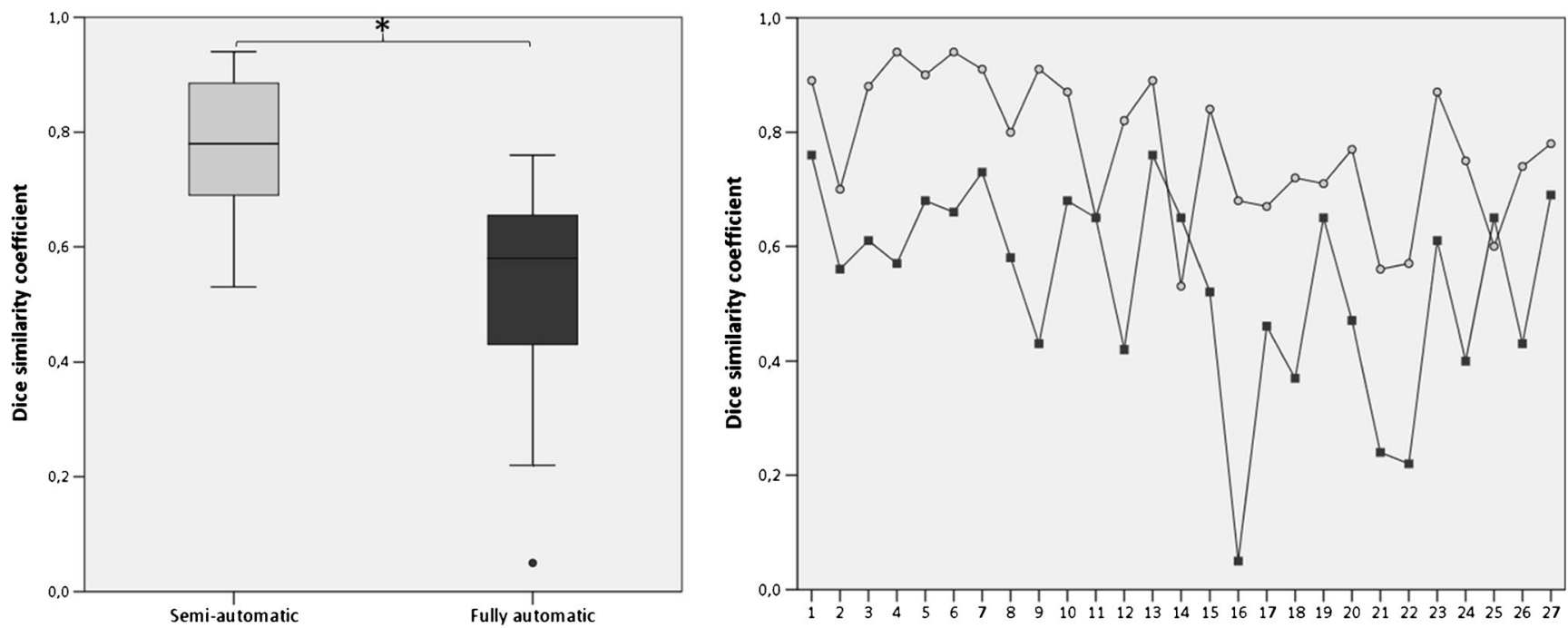

Fig. 3 DSC for semi-automatic (light) and fully automatic (dark) approach (*signif. $p<0.001)$ 


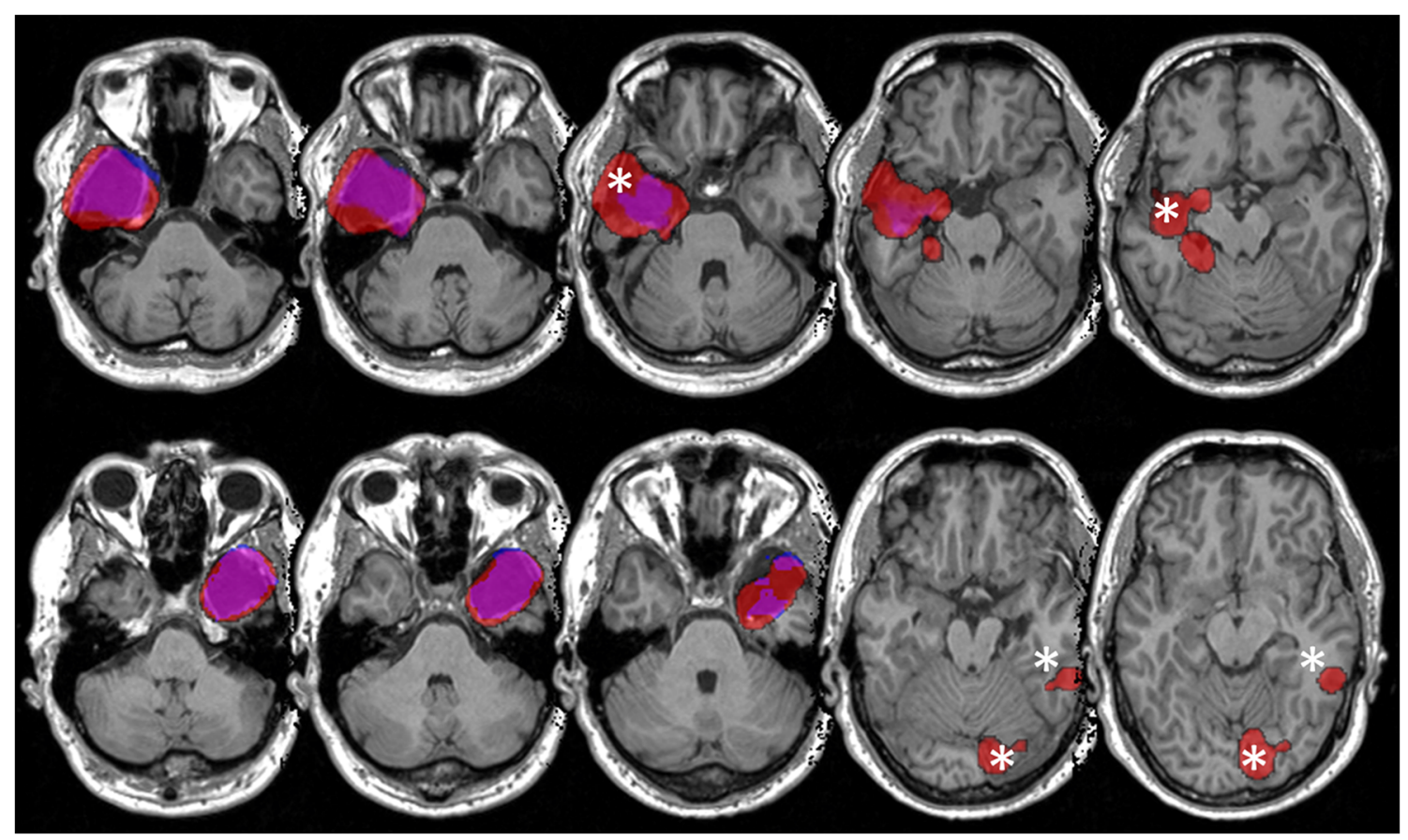

Fig. 4 Individual false-positive results in automatic processing. GNB classifier results (red) are superimposed onto manual segmentation (blue). Areas of congruence are purple. Asterisk (*) indicates areas of false-positive values

0.44 , range $0.14-1.85)$ similarly outperformed $(p<0.001)$ the fully automatic approach (1.32, range 0.42-7.25; Fig. 6-left panel).

To assess the impact of actual lesion size on accuracy, all cases were dichotomized into groups by their median resection volume obtained from manual delineation (median $=$ $17.920 \mathrm{ml}$ ), resulting in 14 large (mean volume $26.02 \pm$ $8.06 \mathrm{ml}$, range $17.92-48.23 \mathrm{ml}$ ) and 13 small resection areas (mean volume $7.39 \pm 3.08 \mathrm{ml}$, range $2.22-12.36 \mathrm{ml}$ ).

As shown in Fig. 7 (left panel) and Table 2, larger resections were associated with significantly better DSCs than smaller resections for both methods $(p<0.05)$. Additionally, resection size was significantly correlated with the DSCs in both semi- and fully automatic approaches $\left(r_{\mathrm{s}}=0.71, p=\right.$ 0.001 vs. $\left.r_{\mathrm{s}}=0.54, p=0.004\right)$. Comparing spatial similarity with aHD, we found no significant difference between the performance in small and large resection areas in both approaches, respectively (Fig. 6, right panel and Table 3).

The accuracy of the semi-automatic approach however significantly outperformed the fully automatic approach irrespective of the resection size $(p<0.001$ for DSC, $p<0.05$ for aHD).

The median PVD with reference to manual segmentation was $0.04(\mathrm{SD} \pm 21.57$; range $-67.18-35.11)$ for the semiautomatic and $-3.13(\mathrm{SD} \pm 61.75$; range $-229.32-41.37)$ for the fully automatic approaches. There was no significant difference between the approaches $(p>0.05)$. In small resections, the PVD of the semi-automatic approach was significantly better than the PVD of the fully automatic approach $(p<0.05, z=-2.691)$, whereas in large resections, there is no significant difference (Table 4 ). The PVDs of the semiautomatic approach did not reveal a significant difference
Fig. 5 Individual performance of all approaches in patient 16 (axial slices)

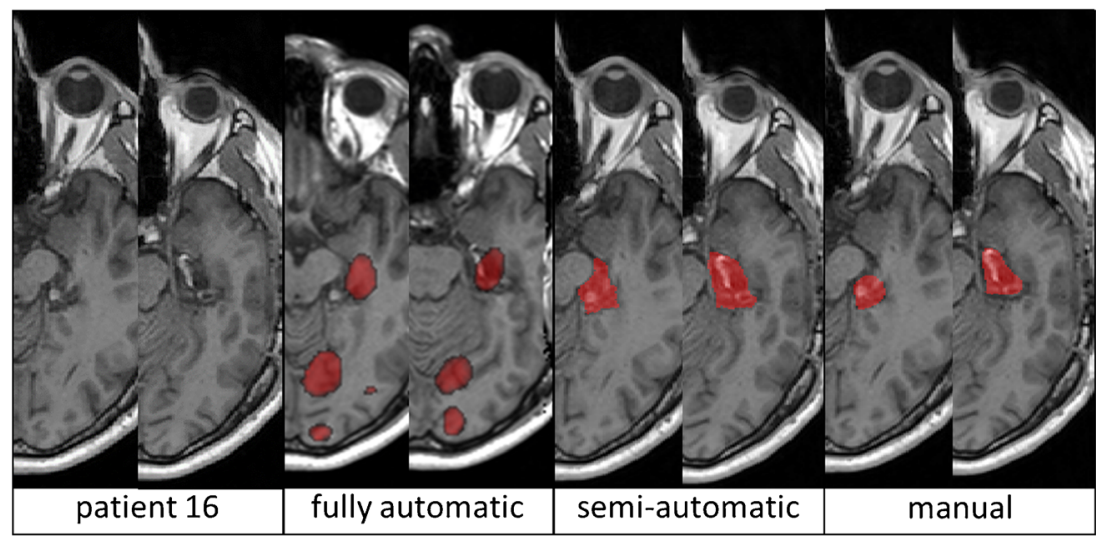



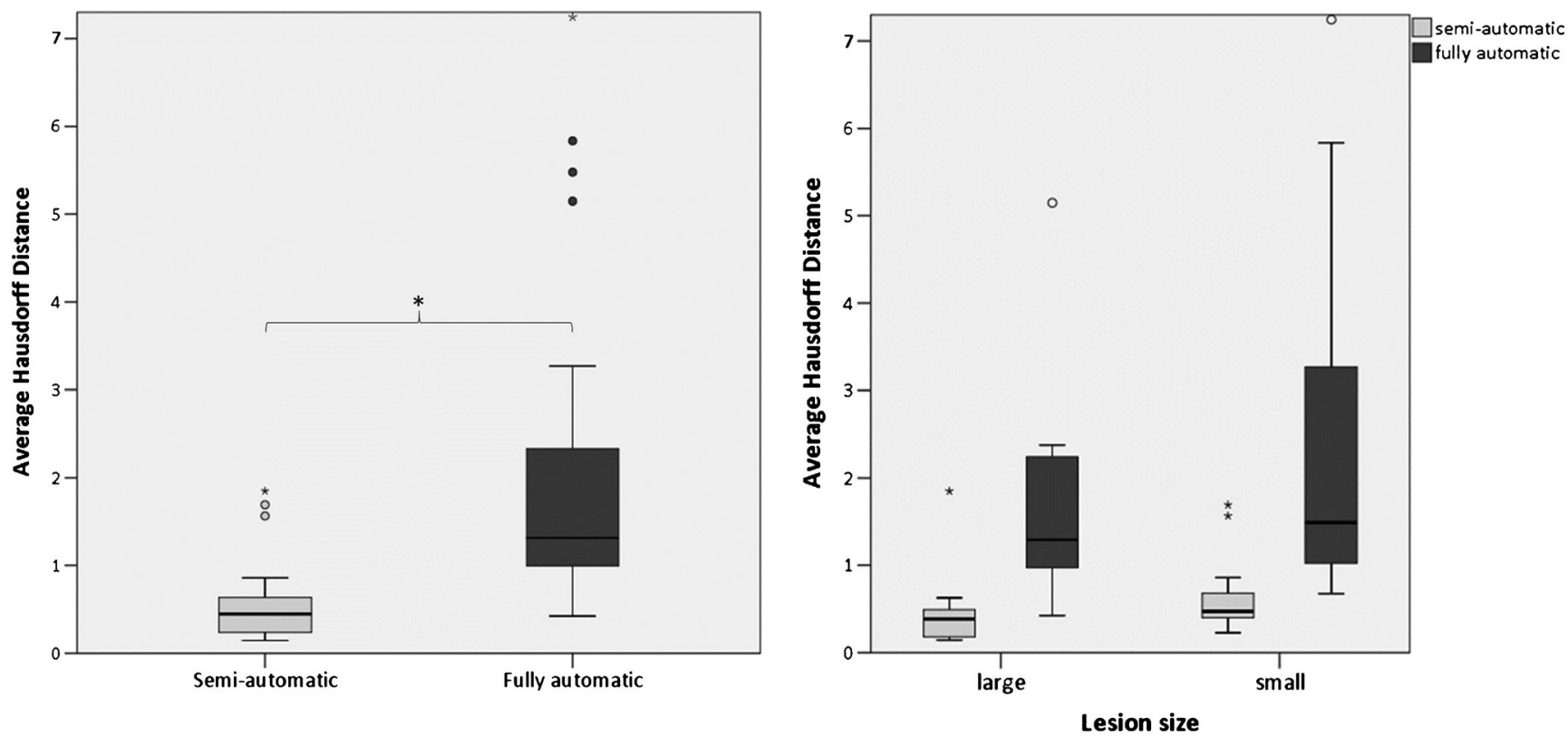

Fig. 6 Averaged Hausdorff distance for semi-automatic (light) and fully automatic (dark) approaches (*signif. $p<0.001$ )

regarding small or large resections, while in the fully automatic approach, the median PVDs significantly differed between the groups $(p<0.05, z=-3.057)$, with better performance in large resections.

\section{Effort and cost}

The average time needed for manual segmentation was $30.41 \mathrm{~min} / \mathrm{subject}(\mathrm{SD} \pm 6.43$, range $17-50 \mathrm{~min}$ ). Userguided semi-automatic lesion segmentation took an average of $3.27 \mathrm{~min} / \mathrm{subject}(\mathrm{SD} \pm 0.96$, range $1.33-5.53 \mathrm{~min}$ ), including manual correction for inaccuracies. The differences in effort between manual and semi-automatic as well as manual and fully automatic or semi-automatic and fully automatic approaches were highly significant at $p<0.001$, respectively.

Importantly, it must be mentioned that the fully automatic approach required a certain amount of processing time on our computational environment, which was $13.41 \mathrm{~min} / \mathrm{subject}$ (SD \pm 2.14 , range 9-19). This was however irrelevant for cost calculation, because this step did not require supervision and the main financial burden stems from personnel cost.

Then, we made a digression into the cost factor of this study. For manual segmentation, an expert rater was needed, whereby at least a postdoctoral researcher is meant (standard salary $6000 € /$ month, $34.62 € / \mathrm{h}$ ). Hence, the mean cost was $17.64 € /$ per subject. To ensure an optimal outcome of the
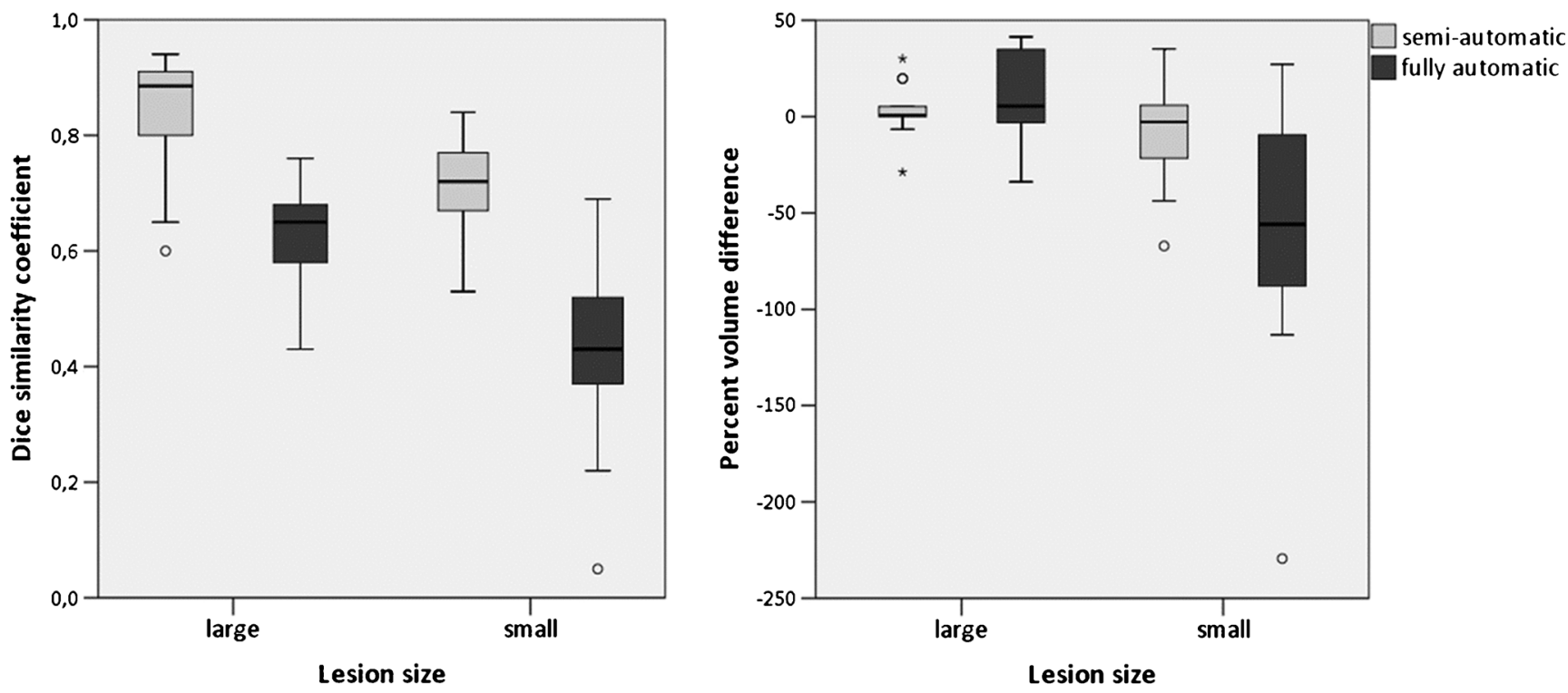

Fig. 7 DSC (left) and PVD (right) of each approach in large and small lesions 
Table 2 Median DSCs of semiand fully automatic segmentations in small and large lesions

\begin{tabular}{llll}
\hline Method & Lesion size & Median DSC & Sign. \\
\hline Semi-automatic & Large & $0.89(\mathrm{SD} \pm 0.11$, range $0.60-0.94)$ & $p<0.05$, \\
& Small & $0.72(\mathrm{SD} \pm 0.10$, range $0.53-0.84)$ & $z=-2.962$ \\
Fully automatic & Large & $0.65(\mathrm{SD} \pm 0.09$, range $0.43-0.76)$ & $p<0.05$, \\
& Small & $0.43(\mathrm{SD} \pm 0.18$, range $0.05-0.69)$ & $z=-3.039$ \\
\hline
\end{tabular}

manual corrections, an expert rater would be equally required for the semi-automatic approach. For this approach, an average of $1.90 € /$ subject had to be invested. The fully automatic segmentation with GNB required no human input for the image segmentation. In that case, mean cost was approaching zero, if image loading steps are disregarded.

\section{Discussion}

This study compared manual to semi- and fully automatic methods for segmentation of resected brain areas on highresolution T1w images. One of the most important questions concerning medical image segmentation is accuracy. We investigated quality and validity of the applied methods using manual segmentations as ground truth. Measures used were the DSC as a global measure of overlap, the aHD as a metric of spatial distance, and PVD for volume agreement, because our intention was to focus on few but relevant parameters which are straightforward to obtain and interpret. The DSC constitutes a robust measure of overlap and has been widely used in the validation of other methods for lesion segmentation [17, 42, 44]. It takes both false positives and false negatives into account and therefore represents a robust performance indicator.

Analyses were completed by the aHD, which is especially recommended when the accuracy of the boundary delineation of the segmentation method is of importance, as it is the case in TLE surgery [47]. However, conventional HD is sensitive to and over-penalizes outliers, which are very likely in the comparison with surgical segmentations [47]. Average HD overcomes these limitations and is therefore particularly well-suited for anatomical image analyses [50]. Irrespective of the used metric, results must be still interpreted with caution - despite generally favorable global aHD, there could still be considerable local disagreement within a complex structure [49].

Table 3 Median aHDs of semiand fully automatic segmentations in small and large lesions
The semi-automatic approach implemented in the ITKSNAP outperformed the fully automatic method independent of lesion size and achieved excellent accuracy (median DSC of 0.78 , median aHD 0.44 ). The high signal intensity contrast between the surgical defect zone and brain tissue as well as the well-defined resection boundaries may have contributed positively to this good performance.

Corroborating previous studies, we found a strong influence of individual lesion size on the DSC [20]. Expectably, the segmentation performance using either approaches was significantly better in larger resections, as the DSC is sensitive to the size of the segmented area [49]. Segmentation errors were indeed mostly located at the boundary and could therefore bias the analysis towards larger segments. Results of the spatial distance analysis support this hypothesis, because it is independent of the size of the segmented area [51].

Focusing on the cases with limited performance of the semiautomatic approach (7 patients with a DSC <0.7), we found interesting commonalities beside lesion size. In three patients (P14, P16, P25) it was difficult to separate the resected area from the enlarged inferior horn of the lateral ventricles. Three resection cavities (P16, P17, P21) were inhomogeneous, and the resection boundaries were not clearly apparent. Segmentation with the ITK-SNAP is relatively robust and in these cases even turned out to be significantly more reproducible than manual segmentation [16]. It can be performed by any rater with experience in manual segmentation [41]. Additionally, placement of the seeds in the ROI does not represent a crucial step per se, as the evolving contour expands in all directions in regions where the speed function is positive and contracts where the speed function is negative. We decided to use the ITK-SNAP due to its simple handling, the evolving visualization of the $3 \mathrm{D}$ volume in real time, and the option to post-process the segmentation using integrated 3D manipulation tools.

The automatic algorithm delivered less satisfactory results (median DSC 0.58, median aHD 1.32) and did not detect the resection

\begin{tabular}{llll}
\hline Method & Lesion size & Median aHD & Sign. \\
\hline Semi-automatic & Large & $0.38(\mathrm{SD} \pm 0.45$, range $0.14-1.85)$ & $p>0.05$ \\
& Small & $0.47(\mathrm{SD} \pm 0.47$, range $0.22-1.69)$ & \\
Fully automatic & Large & $1.29(\mathrm{SD} \pm 1.22$, range $0.42-5.15)$ & $p>0.05$ \\
& Small & $1.49(\mathrm{SD} \pm 2.21$, range $0.67-7.25)$ & \\
\hline
\end{tabular}


Table 4 Median PVDs of semiand fully automatic segmentations in small and large lesions

\begin{tabular}{llll}
\hline Method & Lesion size & Median PVD & Sign. \\
\hline Semi-automatic & Large & $0.72(\mathrm{SD} \pm 13.71$, range $-28.91-29.96)$ & $p>0.05$ \\
& Small & $-2.75(\mathrm{SD} \pm 27.26$, range $-67.18-35.11)$ & \\
Fully automatic & Large & $5.44(\mathrm{SD} \pm 23.63$, range $-33.97-41.37)$ & $p<0.05$, \\
& Small & $-55.88(\mathrm{SD} \pm 70.33$, range $-229.32-27.04)$ & $z=-3.057$ \\
& & & \\
\hline
\end{tabular}

in a single subject, where it detected only parts of the resected area (Fig. 4). It should however be taken into consideration that the resected area was difficult to segregate from surrounding tissue, as itcontained blood debris [52]. This is in line with previous studies which demonstrated reduced precision when tissue signal intensities were similar or the target structure itself was altered [15,21].

This signifies a clear advantage of the semi-automatic approach, as the manual interaction described above ensures satisfying results even in cases were other methods might fail. It has however to be emphasized that the GNB classifier was trained on stroke lesions, and while both surgical resection cavities and resorbed lesioned tissue in chronic stroke will ultimately contain CSF, there are still differences in signal intensity. Nevertheless, no segmentation algorithm specifically designed for neurosurgical resections has been proposed to date, necessitating use of established methods.

Notwithstanding expectable limitations, selection of the lesion GNB was driven by several potential advantages: It operates on unimodal $\mathrm{T} 1 \mathrm{w}$ data, requires no control population or arbitrary thresholding, and integrates with SPM, utilizing default segmentation and normalization routines [44]. This precluded consideration of other algorithms from the ischemic stroke lesion segmentation (ISLES) challenge [53], as they require either multispectral data [41, 42] or healthy control groups [13], which might not be readily available in all research environments. While our results revealed reduced accuracy, implementation of TLE-specific training datasets into the lesion_GNB would clearly improve lesion detection. This was however beyond the scope of this study and will be addressed in future work.

As a second point, we assessed practical aspects of the different segmentation methods. Regarding practical implementation in research environments, we relied on freely available software and standard computational hardware. As previous studies emphasized, manual segmentation is extremely tedious and, depending on lesion complexity, very timeconsuming and thus expensive [13, 20, 54]. Manual segmentation in our study required an average human input of $30.41 \mathrm{~min} /$ subject, mainly due to the uniformity of TLE resections. Manual tracing furthermore requires sound anatomical knowledge, at least at the level of postdoctoral research. For larger cohorts, manual segmentation would therefore implicate a tremendous amount of manual work and cost.

The semi-automatic method requires similar expertise; it was however significantly faster compared to manual tracing $(3.27 \mathrm{~min} /$ subject), resulting in a cost reduction by 15.74 Euro per subject. In this regard, fully automatic segmentation methods could offer even better cost-effectiveness. They are less time-consuming and do not require an expert as they can be applied by individuals with limited experience [44]. Interaction during processing is minimal, resulting in cost per subject approaching zero. The lesion_GNB algorithm performed well on standard computers (13.41 min/subject), whereas availability of high-performance computational equipment would further increase throughput.

\section{Conclusion}

Our findings suggest that semi-automatic methods are currently most efficient for the segmentation of surgical resections. They offer the best compromise between precision and effort, which is particularly relevant for the evaluation of larger cohorts. Its superior accuracy compared to the automatic method proved that human input can further improve computerized segmentations.

\section{Limitations}

No formal assessment of inter-rater reliability in the manual or semi-automatic approaches was attempted here as this was extensively explored in previous studies [55]. Although the cohort was small, the sample size was sufficient to demonstrate significant differences between the approaches. Moreover, although intraclass correlation coefficients (ICC) are often used to compare segmentation techniques, the ICC strongly depends on sample size and distribution of subjects, especially in smaller cohorts [56], thus precluding use of ICC as a measure of accuracy in our study.

Acknowledgements Open Access funding provided by Projekt DEAL.

Funding information NAF is the recipient of a research grant awarded by the German Research Foundation (DFG, FO996/1-1).

\section{Compliance with ethical standards}

Conflict of interest The authors declare that they have no conflict of interest.

Ethical approval Institutional Review Board approval was obtained for this study. All procedures performed in this study were in accordance with the ethical standards of the institutional research committee and/or national research committee and with the 1964 Helsinki Declaration and its later amendments or comparable ethical standards. 
Informed consent Individual informed consent was waived for this study.

Open Access This article is licensed under a Creative Commons Attribution 4.0 International License, which permits use, sharing, adaptation, distribution and reproduction in any medium or format, as long as you give appropriate credit to the original author(s) and the source, provide a link to the Creative Commons licence, and indicate if changes were made. The images or other third party material in this article are included in the article's Creative Commons licence, unless indicated otherwise in a credit line to the material. If material is not included in the article's Creative Commons licence and your intended use is not permitted by statutory regulation or exceeds the permitted use, you will need to obtain permission directly from the copyright holder. To view a copy of this licence, visit http://creativecommons.org/licenses/by/4.0/.

\section{References}

1. Bates E, Wilson SM, Saygin AP, Dick F, Sereno MI, Knight RT, Dronkers NF (2003) Voxel-based lesion-symptom mapping. Nat Neurosci 6:448-450. https://doi.org/10.1038/nn1050

2. Vaidya AR, Pujara MS, Petrides M, Murray EA, Fellows LK (2019) Lesion studies in contemporary neuroscience. Trends Cogn Sci 23:653-671. https://doi.org/10.1016/j.tics.2019.05.009

3. Karnath H-O, Sperber C, Rorden C (2018) Mapping human brain lesions and their functional consequences. NeuroImage 165:180 189. https://doi.org/10.1016/j.neuroimage.2017.10.028

4. Wang Y, Qian T, You G, Peng X, Chen C, You Y, Yao K, Wu C, Ma J, Sha Z, Wang S, Jiang T (2015) Localizing seizure-susceptible brain regions associated with low-grade gliomas using voxel-based lesion-symptom mapping. Neuro-Oncol 17:282-288. https://doi. org/10.1093/neuonc/nou130

5. Rorden C, Fridriksson J, Karnath H-O (2009) An evaluation of traditional and novel tools for lesion behavior mapping. NeuroImage 44:1355-1362. https://doi.org/10.1016/j.neuroimage. 2008.09.031

6. Rudrauf D, Mehta S, Bruss J, Tranel D, Damasio H, Grabowski TJ (2008) Thresholding lesion overlap difference maps: application to category-related naming and recognition deficits. NeuroImage 41: 970-984. https://doi.org/10.1016/j.neuroimage.2007.12.033

7. Gleichgerrcht E, Fridriksson J, Rorden C, Bonilha L (2017) Connectome-based lesion-symptom mapping (CLSM): a novel approach to map neurological function. NeuroImage Clin 16:461467. https://doi.org/10.1016/j.nicl.2017.08.018

8. Wawrzyniak M, Klingbeil J, Zeller D, Saur D, Classen J (2018) The neuronal network involved in self-attribution of an artificial hand: a lesion network-symptom-mapping study. NeuroImage 166:317324. https://doi.org/10.1016/j.neuroimage.2017.11.011

9. Fiez JA, Damasio H, Grabowski TJ (2000) Lesion segmentation and manual warping to a reference brain: intra- and interobserver reliability. Hum Brain Mapp 9:192-211. https://doi.org/10.1002/ (SICI)1097-0193(200004)9:4<192::AID-HBM2>3.0.CO;2-Y

10. Deeley MA, Chen A, Datteri R, Noble JH, Cmelak AJ, Donnelly EF, Malcolm AW, Moretti L, Jaboin J, Niermann K, Yang ES, Yu DS, Yei F, Koyama T, Ding GX, Dawant BM (2011) Comparison of manual and automatic segmentation methods for brain structures in the presence of space-occupying lesions: a multi-expert study. Phys Med Biol 56:4557-4577. https://doi.org/10.1088/0031-9155/ $56 / 14 / 021$

11. Farr TD, Wegener S (2010) Use of magnetic resonance imaging to predict outcome after stroke: a review of experimental and clinical evidence. J Cereb Blood Flow Metab 30:703-717. https://doi.org/ $10.1038 / \mathrm{jcbfm} .2010 .5$
12. Gryska EA, Schneiderman J, Heckemann RA (2019) Automatic brain lesion segmentation on standard MRIs of the human head: a scoping review protocol. BMJ Open 9:e024824. https://doi.org/10. 1136/bmjopen-2018-024824

13. Seghier ML, Ramlackhansingh A, Crinion J, Leff AP, Price CJ (2008) Lesion identification using unified segmentationnormalisation models and fuzzy clustering. Neuroimage 41:12531266. https://doi.org/10.1016/j.neuroimage.2008.03.028

14. Egger C, Opfer R, Wang C, Kepp T, Sormani MP, Spies L, Barnett M, Schippling S (2016) MRI FLAIR lesion segmentation in multiple sclerosis: does automated segmentation hold up with manual annotation? NeuroImage Clin 13:264-270. https://doi.org/10.1016/ j.nicl.2016.11.020

15. de Haan B, Clas P, Juenger H, Wilke M, Karnath HO (2015) Fast semi-automated lesion demarcation in stroke. NeuroImage Clin 9: 69-74. https://doi.org/10.1016/j.nicl.2015.06.013

16. Yushkevich PA, Piven J, Hazlett HC, Smith RG, Ho S, Gee JC, Gerig G (2006) User-guided 3D active contour segmentation of anatomical structures: significantly improved efficiency and reliability. NeuroImage 31:1116-1128 16/j.neuroimage.2006.01.015

17. Maier O, Wilms M, von der Gablentz J, et al (2014) Ischemic stroke lesion segmentation in multi-spectral MR images with support vector machine classifiers. pp 903504-903504-12

18. Kim H, Mansi T, Bernasconi N, Bernasconi A (2012) Surfacebased multi-template automated hippocampal segmentation: application to temporal lobe epilepsy. Med Image Anal 16:1445-1455. https://doi.org/10.1016/j.media.2012.04.008

19. Shen S, Szameitat AJ, Sterr A (2010) An improved lesion detection approach based on similarity measurement between fuzzy intensity segmentation and spatial probability maps. Magn Reson Imaging 28:245-254. https://doi.org/10.1016/j.mri.2009.06.007

20. Wilke M, de Haan B, Juenger H, Karnath H-O (2011) Manual, semi-automated, and automated delineation of chronic brain lesions: a comparison of methods. NeuroImage 56:2038-2046. https://doi.org/10.1016/j.neuroimage.2011.04.014

21. Guo D, Fridriksson J, Fillmore P, Rorden C, Yu H, Zheng K, Wang S (2015) Automated lesion detection on MRI scans using combined unsupervised and supervised methods. BMC Med Imaging:15. https://doi.org/10.1186/s12880-015-0092-x

22. Rudie JD, Colby JB, Salamon N (2015) Machine learning classification of mesial temporal sclerosis in epilepsy patients. Epilepsy Res 117:63-69. https://doi.org/10.1016/j.eplepsyres.2015.09.005

23. Deo RC (2015) Machine learning in medicine. Circulation 132: 1920-1930. https://doi.org/10.1161/CIRCULATIONAHA.115. 001593

24. Gillebert CR, Humphreys GW, Mantini D (2014) Automated delineation of stroke lesions using brain CT images. NeuroImage Clin 4:540-548. https://doi.org/10.1016/j.nicl.2014.03.009

25. Sanjuan A, Price CJ, Mancini L et al (2013) Automated identification of brain tumors from single MR images based on segmentation with refined patient-specific priors. Front Neurosci:7. https://doi. org/10.3389/fnins.2013.00241

26. Stamatakis EA, Tyler LK (2005) Identifying lesions on structural brain images - validation of the method and application to neuropsychological patients. Brain Lang 94:167-177. https://doi.org/10. 1016/j.bandl.2004.12.010

27. Choy G, Khalilzadeh O, Michalski M et al (2018) Current applications and future impact of machine learning in radiology. Radiology 288:318-328. https://doi.org/10.1148/radiol.2018171820

28. Handelman GS, Kok HK, Chandra RV, Razavi AH, Huang S, Brooks M, Lee MJ, Asadi H (2019) Peering into the black box of artificial intelligence: evaluation metrics of machine learning methods. AJR Am J Roentgenol 212:38-43. https://doi.org/10. 2214/AJR.18.20224

29. Sagher O, Thawani JP, Etame AB, Gomez-Hassan DM (2012) Seizure outcomes and mesial resection volumes following selective 
amygdalohippocampectomy and temporal lobectomy. Neurosurg Focus 32:E8. https://doi.org/10.3171/2011.12.FOCUS11342

30. Fernandes DA, Yasuda CL, Lopes TM, Enrico G, Alessio A, Tedeschi H, de Oliveira E, Cendes F (2014) Long-term postoperative atrophy of contralateral hippocampus and cognitive function in unilateral refractory MTLE with unilateral hippocampal sclerosis. Epilepsy Behav 36:108-114. https://doi.org/10.1016/j.yebeh.2014. 04.028

31. Gaça LB, Garcia MTFC, Sandim GB, Assumption Leme IB, Noffs MHS, Carrete Júnior H, Centeno RS, Sato JR, Yacubian EMT (2018) Morphometric MRI features and surgical outcome in patients with epilepsy related to hippocampal sclerosis and low intellectual quotient. Epilepsy Behav 82:144-149. https://doi.org/10. 1016/j.yebeh.2018.03.011

32. Gross RE, Willie JT, Drane DL (2016) The role of stereotactic laser amygdalohippocampotomy in mesial temporal lobe epilepsy. Neurosurg Clin N Am 27:37-50. https://doi.org/10.1016/j.nec. 2015.08.004

33. Gleichgerrcht E, Bonilha L, Qiu D et al (2019) Mapping the neural basis of declarative verbal memory: evidence from mesial temporal lobe lesions (P3.1-014). Neurology 92:P3.1-P014

34. Drane DL, Loring DW, Voets NL, Price M, Ojemann JG, Willie JT, Saindane AM, Phatak V, Ivanisevic M, Millis S, Helmers SL, Miller JW, Meador KJ, Gross RE (2015) Better object recognition and naming outcome with MRI-guided stereotactic laser amygdalohippocampotomy for temporal lobe epilepsy. Epilepsia 56:101-113. https://doi.org/10.1111/epi.12860

35. Yogarajah M, Focke NK, Bonelli SB, Thompson P, Vollmar C, McEvoy AW, Alexander DC, Symms MR, Koepp MJ, Duncan JS (2010) The structural plasticity of white matter networks following anterior temporal lobe resection. Brain 133:2348-2364

36. Andersen SM, Rapcsak SZ, Beeson PM (2010) Cost function masking during normalization of brains with focal lesions: still a necessity? NeuroImage 53:78-84. https://doi.org/10.1016/j. neuroimage.2010.06.003

37. Wagner J, Urbach H, Niehusmann P, von Lehe M, Elger CE, Wellmer J (2011) Focal cortical dysplasia type IIb: completeness of cortical, not subcortical, resection is necessary for seizure freedom. Epilepsia 52:1418-1424. https://doi.org/10.1111/j.15281167.2011.03158.x

38. Yushkevich PA, Pashchinskiy A, Oguz I, Mohan S, Schmitt JE, Stein JM, Zukić D, Vicory J, McCormick M, Yushkevich N, Schwartz N, Gao Y, Gerig G (2019) User-guided segmentation of multi-modality medical imaging datasets with ITK-SNAP. Neuroinformatics 17:83-102. https://doi.org/10.1007/s12021-0189385-x

39. Zhu SC, Yuille AL (1995) Region competition and its analysis: a unified theory for image segmentation

40. Caselles V, Kimmel R, Sapiro G (1997) Geodesic active contours. Int J Comput Vis 22:61-79. https://doi.org/10.1023/A: 1007979827043

41. Yushkevich PA, Gao Y, Gerig G (2016) ITK-SNAP: an interactive tool for semi-automatic segmentation of multi-modality biomedical images. In: 2016 38th Annual International Conference of the IEEE Engineering in Medicine and Biology Society (EMBC). pp 3342 3345

42. Fyllingen EH, Stensjøen AL, Berntsen EM, Solheim O, Reinertsen I (2016) Glioblastoma segmentation: comparison of three different software packages. PLoS One 11:e0164891. https://doi.org/10. 1371/journal.pone. 0164891

43. Yushkevich PA, Gerig G (2017) ITK-SNAP: an interactive medical image segmentation tool to meet the need for expert-guided segmentation of complex medical images. IEEE Pulse 8:54-57. https://doi.org/10.1109/MPUL.2017.2701493

44. Griffis JC, Allendorfer JB, Szaflarski JP (2016) Voxel-based Gaussian naïve Bayes classification of ischemic stroke lesions in individual T1-weighted MRI scans. J Neurosci Methods 257:97108. https://doi.org/10.1016/j.jneumeth.2015.09.019

45. Ito KL, Kim H, Liew S-L (2019) A comparison of automated lesion segmentation approaches for chronic stroke T1-weighted MRI data. Hum Brain Mapp 40:4669-4685. https://doi.org/10.1002/hbm. 24729

46. Zou KH, Warfield SK, Bharatha A, Tempany CMC, Kaus MR, Haker SJ, Wells WM III, Jolesz FA, Kikinis R (2004) Statistical validation of image segmentation quality based on a spatial overlap index. Acad Radiol 11:178-189. https://doi.org/10.1016/S10766332(03)00671-8

47. Taha AA, Hanbury A (2015) Metrics for evaluating 3D medical image segmentation: analysis, selection, and tool. BMC Med Imaging 15. https://doi.org/10.1186/s12880-015-0068-x

48. Cárdenes R, de Luis-García R, Bach-Cuadra M (2009) A multidimensional segmentation evaluation for medical image data. Comput Methods Prog Biomed 96:108-124. https://doi.org/10. 1016/j.cmpb.2009.04.009

49. Kim HS, Park SB, Lo SS, Monroe JI, Sohn JW (2012) Bidirectional local distance measure for comparing segmentations: bidirectional local distance measure. Med Phys 39:6779-6790. https://doi.org/ $10.1118 / 1.4754802$

50. Taha AA, Hanbury A, del Toro OAJ (2014) A formal method for selecting evaluation metrics for image segmentation. In: 2014 IEEE International Conference on Image Processing (ICIP). IEEE, Paris, pp 932-936

51. Khotanlou H, Colliot O, Atif J, Bloch I (2009) 3D brain tumor segmentation in MRI using fuzzy classification, symmetry analysis and spatially constrained deformable models. Fuzzy Sets Syst 160: 1457-1473. https://doi.org/10.1016/j.fss.2008.11.016

52. Herrmann E, Ermis E, Jungo A et al (2018) P01.088 brain resection cavity delineation for radiation target volume definition in glioblastoma patients using deep learning. Neuro-Oncol 20:iii250-iii251. https://doi.org/10.1093/neuonc/noy139.130

53. Winzeck S, Hakim A, McKinley R et al (2018) ISLES 2016 and 2017-Benchmarking Ischemic Stroke Lesion Outcome Prediction Based on Multispectral MRI. Front Neurol 9. https://doi.org/10. 3389/fneur.2018.00679

54. Pustina D, Coslett HB, Turkeltaub PE, Tustison N, Schwartz MF, Avants B (2016) Automated segmentation of chronic stroke lesions using LINDA: lesion identification with neighborhood data analysis. Hum Brain Mapp 37:1405-1421. https://doi.org/10.1002/hbm. 23110

55. Despotović I, Goossens B, Philips W (2015) MRI segmentation of the human brain: challenges, methods, and applications. In: Comput. Math. Methods Med. https://www.hindawi.com/journals/ cmmm/2015/450341/. Accessed 26 Jul 2018

56. Mehta S, Bastero-Caballero RF, Sun Y, Zhu R, Murphy DK, Hardas B, Koch G (2018) Performance of intraclass correlation coefficient (ICC) as a reliability index under various distributions in scale reliability studies. Stat Med 37:2734-2752. https://doi.org/ $10.1002 / \operatorname{sim} .7679$

Publisher's note Springer Nature remains neutral with regard to jurisdictional claims in published maps and institutional affiliations. 